\title{
Revista Ciencias de la Salud
}

\section{Artículo}

Aportes de TIC's en Práctica Digital de la Salud para el abordaje oportuno de la persona con Cáncer en estadio terminal desde el punto de vista de la Rehabilitación

"Contributions of ICTs in Digital Health Practice for the timely embedding of the person with terminal Cancer from the point of view of Rehabilitation

\section{Autores:}

Dra. María Gabriela Araya Medrano

2 Investigador independiente

correo;gatf03@gmail.com

Palabras clave: Neoplasia, Telemedicina, Fisioterapia, Modalidades de fisioterapia, Medicina Física, ECNT

\section{DOl: $10.54376 /$ rcmui.v1i1.99}

\section{RESUMEN}

La pandemia mundial causada por el COVID-19 ha dado auge al Teletrabajo en casi todos los sectores, incluyendo la atención en servicios de Salud. La implementación del Tecnologías de la Información y la Comunicación Tecnologías de la Información y de la Comunicación (TIC's) en Telemedicina parece ser una herramienta prometedora la Rehabilitación Oncológica en personas con cáncer en estadio terminal. Objetivo: Investigar los alcances de las TIC's en Práctica Digital de la Salud orientada a la Rehabilitación de la persona con Cáncer en estadio terminal. Materiales y métodos: Estudio descriptivo de revisión bibliográfica. Se consultan recursos digitales como PubMed, LILACS, SciELO, Elsevier, RefSeek, Google Académico, Dialnet, Academia, Base, Springer Link, ERIC y Redalyc como fuente de documentos publicados (libros, tesis, artículos 
científicos). Se utilizan los descriptores Neoplasia AND Telemedicina AND Fisioterapia OR Modalidades de Fisioterapia OR Medicina Física y Rehabilitación OR Rehabilitación AND Cuidados Paliativos. Se incluyen documentos publicados durante los últimos 20 años, en español, inglés y portugués. Considerando la evidencia publicada, se establece los posibles alcances de las TIC's en Telesalud en las personas con cáncer terminal. Resultados: Se realiza una Análisis FODA de la bibliografía encontrada y se brindan recomendaciones de posibles adaptaciones para el uso de las TIC's en Telesalud en las personas con cáncer terminal Conclusión: El uso de las TIC's en salud y rehabilitación en otras situaciones de salud parece brindar aportes favorecedores para todos los implicados, y generar resultados similares a la atención presencial, sin embargo, no debe sustituirla si no ser un apoyo. La evidencia científica sobre el tema estudiado es escasa, se debe realizar más investigación y publicar los resultados de las TIC's en Telesalud en las personas con cáncer terminal.

\section{ABSTRACT}

The global pandemic caused by COVID-19 has given rise to Teleworking in almost all sectors, including healthcare services. The implementation of Information and Communication Technologies Information and Communication Technologies (ICTs) in Telemedicine seems to be a promising tool for Oncological Rehabilitation in people with terminal stage cancer. Objective: To investigate the scope of ICTs in Digital Health Practice aimed at the Rehabilitation of the person with terminal Cancer. Materials and methods: Descriptive study of bibliographic review. Digital resources such as PubMed, LILACS, SciELO, Elsevier, RefSeek, Google Academic, Dialnet, Academia, Base, Springer Link, ERIC and Redalyc are consulted as a source of published documents (books, theses, scientific articles). The descriptors Neoplasia AND Telemedicine AND Physiotherapy OR Physiotherapy Modalities OR Physical Medicine and Rehabilitation OR Rehabilitation AND Palliative Care are used. Documents published during the last 20 years are included, in Spanish, English and Portuguese. Considering the published evidence, the possible scope of ICTs in Telehealth in people with terminal cancer is established. Results: A FODA Analysis of the bibliography found is carried out and recommendations are provided for possible adaptations for the use of ICTs in Telehealth in people with terminal cancer Conclusion: The use of ICTs in health and rehabilitation in other health situations seems to provide favorable contributions for all those involved, and generate results similar to face-to-face care, however, it should not replace it if it is not a support. The scientific evidence on the subject studied is scarce, more research should be carried out and the results of ICTs in Telehealth published in people with terminal cancer. 


\section{people with terminal cancer.}

Introducción

A nivel mundial el cáncer es la segunda causa de morbilidad y mortalidad dentro del grupo de Enfermedades Crónicas no Transmisibles (ECNT). En Costa Rica, durante las últimas décadas, el grupo de enfermedades cancerígenas, han ido en aumento y ocupan el segundo lugar en la tasa de mortalidad a causa de ECNT, solo superadas por enfermedades cardiovasculares (i). Estas enfermedades muchas veces necesitan de Cuidado Paliativo y Acompañamiento al Final de la Vida.

La Organización Mundial de la Salud (OMS) define la atención paliativa como un enfoque que mejora la calidad de vida de los pacientes y sus familias frente a los problemas asociados con las enfermedades potencialmente mortales, mediante la prevención y el alivio del sufrimiento, y son estos un sistema de apoyo para ayudar a los pacientes a vivir lo más activamente posible hasta la muerte, mitigando el dolor y otros síntomas, y proporcionando apoyo espiritual y psicológico desde el momento del diagnóstico hasta el final de la vida y durante el duelo (ii,iii). En fase terminal del cáncer la aplicación de Cuidados Paliativos es de gran importancia, son estos un sistema de apoyo para ayudar a los pacientes a vivir lo más activamente posible hasta la muerte (3).

En 1980 la OMS promueve el Programa de Cuidados Paliativos como parte del Programa de Control de Cáncer. En Costa Rica inician los Cuidados Paliativos en 1987, ya para los años noventa, los Cuidados Paliativos organizan la atención de enfermos con cáncer terminal, como respuesta a las necesidades físicas, emocionales y espirituales de los pacientes que sufrían de dolor crónico a raíz de cáncer (iv).

De acuerdo con la OMS, se considera a la Telemedicina, como la prestación de servicios de salud, en los que la distancia es un factor determinante, por parte de profesionales sanitarios a través de la utilización de TICs, con el fin de lograr el intercambio de información válida para el diagnóstico, el tratamiento, la prevención de enfermedades, la investigación y la evaluación y para la formación continuada de profesionales sanitarios, todo ello con el objetivo final de mejorar la salud de la población y de las comunidades(v).

El término digital está empezando a reemplazar y abarcar el término "tele", ya que es más representativo de la gama de tecnologías y el impacto que estas tecnologías están teniendo en los modelos de servicios y $\mathrm{s}$ actuales y futuros (vi). Sin embargo, para efectos prácticos, se tomará el vocablo del TIC's en Práctica Digital 
de la Salud para el presente trabajo. Digital es aquella que describe los servicios de atención en salud, el apoyo y la información que se brinda de forma remota a través de dispositivos y comunicación digital cuyo propósito en Fisioterapia y Rehabilitación es facilitar la prestación efectiva de servicios mejorando el acceso a la atención e información y realizando la atención de fisioterapia en salud, a través de estos medios (6).

Aunque las TIC's en Práctica Digital de la Salud se ha desarrollado a principalmente en Europa y Norte América (vii), esta no es una nueva en América Latina. En Costa Rica se impulsan los estudios sobre TIC's en Digital de la Salud desde 1994, producto de la colaboración entre el Ministerio de Salud, el Instituto de Electricidad de Costa Rica y la State Open University. Desde entonces, se ha trabajado en el sector de la TIC's en Práctica Digital de la Salud Hospitalaria.

Sin embargo, esta iniciativa progresa de manera lenta, siendo el componente educativo el más desarrollado en ellas (viii). Gracias a dos pandemias se impulsaron la TIC's en Práctica Digital de la Salud en Costa Rica. Durante el 2009-2010, en medio de la pandemia de gripe AH1N1, se impulsa su uso en conferencias basadas en la TIC's (3). Y en el 2019, debido a la pandemia por el COVID-19, muchos de los servicios de salud han tenido que innovar e introducirse en este ámbito poco desarrollado incluyendo, la Rehabilitación.

La Rehabilitación es un aspecto esencial en la atención integral de las personas con cáncer como parte del cuidado, para disminuir las posibles secuelas físicas y psicológicas, aliviar el dolor, favorecer la reinserciónreintegración a las actividades laborales, sociales y familiares de los pacientes, vivir con dignidad. Tomando en cuenta que los objetivos primordiales del tratamiento del cáncer son curar, prolongar vida y/o mejorar la calidad la vida (ix).

La TIC's en Práctica Digital de la Salud corresponde a un método de prestación de servicios de rehabilitación que utiliza la tecnología para atender a personas, médicos y sistemas, minimizando así las barreras de distancia, tiempo y costo. Es la aplicación de tecnologías de telecomunicación, teledetección y de operación, y tecnologías informáticas para ayudar en la prestación de servicios de rehabilitación médica a distancia (x).

Es necesario ir más allá y explotar todas las ventajas de la TIC's en Práctica Digital de la Salud en las diferentes áreas de la salud y requerimientos que presenta la población ya que no se había escudriñado a profundidad los beneficios que esta modalidad de atención trae consigo en la atención de diversas patologías, entre ellas, Rehabilitación cáncer en fase terminal. 
Las personas con cáncer son más susceptibles de contraer cualquier infección debido a que la enfermedad y tratamientos anticancerígenos (quimioterapia, radioterapia, trasplantes de médula ósea) debilitan las defensas del organismo dando como resultado un estado inmunosupresor (xi). Prueba de esto son los resultados obtenidos por Dignani et al (xii) durante la pandemia del la Influenza (H1N1), los cuales evidencian como la población de personas con cáncer posee una mayor incidencia de neumonía (66\%) y mortalidad a los 30 días del contagio $(18,5 \%)$ comparada con la población general sin cáncer.

En otro estudio reciente sobre COVID-19 con un total de 1.524 pacientes con cáncer, estos pacientes tenían un riesgo dos veces mayor de infección por COVID-19 en comparación con la población general (xiii). Lo anterior se explica a las complicaciones de pacientes con cáncer, incluidas visitas frecuentes al hospital y admisiones (12), estado inmunodeprimido, edad avanzada y mal estado funcional (xiv).

Entre los objetivos del Plan Nacional de Prevención y Atención Integral del Cáncer de Costa Rica, se encuentran aumentar la cobertura en la atención integral del cáncer; mejorar la calidad de los servicios de atención del cáncer; y contribuir a mejorar la calidad de vida de las personas afectadas (1). Uclés et al., menciona, que entre las prioridades y desafíos pendientes del Plan, se encuentran: garantizar el acceso a los servicios de salud de atención integral, desarrollar capacidades y competencias del recurso humano que se adecuen a las necesidades de la población, fortalecer la coordinación y articulación entre instituciones, sectores y sociedad civil, para proteger y mejorar el estado de salud de la población e implementar nuevas tecnologías $(x)$.

Debido a las razones establecidas anteriormente, es de gran importancia la elaboración de trabajos de investigación que desarrollen el tema de TIC's en Práctica Digital de la Salud en Rehabilitación, las cuales demuestren los alcances, limitaciones, ventajas y desventajas de esta, además, insten y guíen a los profesionales en temas tal como el uso de las mismas en Rehabilitación y Cuidados Paliativos y Acompañamiento en Final de Vida en Cáncer en estadio terminal.

\section{Materiales y métodos}

El presente es un estudio de revisión bibliográfica de tipo cualitativo observacional. Se utilizan los buscadores PubMed, LILACS, SciELO, Elseiver, RefSeek, Google Académico, Dialnet, Academia, Base, Springer Link, ERIC y Redalyc. Los descriptores utilizados son Neoplasia AND Telemedicina AND Fisioterapia OR Modalidades de Fisioterapia OR Medicina Física y Rehabilitación OR Rehabilitación AND Cuidados Paliativos. Se 
consideran estudios de casos, metaanálisis, revisiones bibliográficas, artículos en revisión y tesis en inglés, español y portugués entre 2000 y 2020, sin embargo, se consideran documentos de publicación anterior al por relevancia de antigüedad.

Se obtiene de la búsqueda un total de 89 documentos seleccionados de acuerdo a su título, resumen y consideraciones finales con el propósito de valorar la calidad y pertinencia con el tema, descartando 31 documentos duplicados y por criterios de exclusión (estudios con antigüedad de publicación mayor a 20 años; población meta sobrevivientes de cáncer, personas declaradas sanas, que no están en estado terminal y/o en cuidado paliativo), así como artículos de otras enfermedades no relacionadas con el cáncer.

Se leen 58 documentos a cabalidad, descartando 13 debido a los criterios de exclusión. En la figura 1, se puede observar el algoritmo de búsqueda, selección y extracción de la información, además del nivel de evidencia según Sackett aplicado a los documentos relacionados al uso de TIC's en la Práctica Digital de la Salud en Cáncer en estadio terminal.

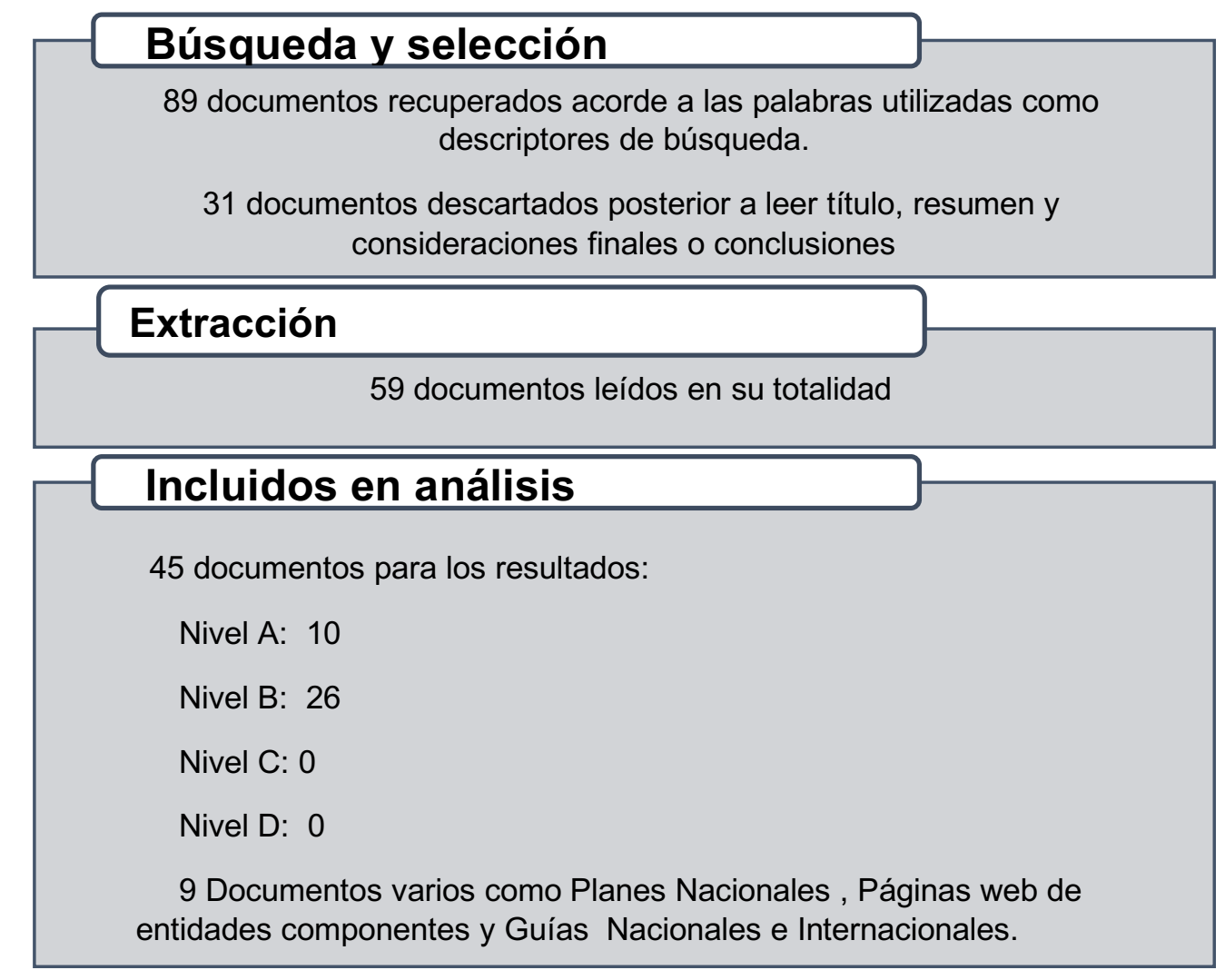

Figura N1 Algoritmo de búsqueda, selección y extracción de la información. 
Fuente: Elaboración propia, 2021.

\section{Resultados}

Se incluyen para los resultados 45 documentos relacionados con TIC's en la Práctica Digital de la Salud en Cáncer en estadio terminal; la figura N1 detalla el flujo de búsqueda, selección y extracción de la información. De acuerdo con los niveles de evidencia según Sackett, los artículos incluidos que se refieren a tratamientos de Telerehabilitación en Cuidado Paliativo en Cáncer en Estadio Terminal fueron: 10 documentos de nivel A, 26 documentos de nivel $B, 0$ documento de nivel $C ; 0$ documentos de nivel D. Además, se incluyeron 1 plan Nacional, 4 página Web de entidades competentes, 4 Guías sobre TIC's en Práctica Digital de la Rehabilitación.

Estos documentos desarrollan temas sobre la aplicación de TIC's en Práctica Digital de la Salud en personas con cáncer en estadio terminal que incluye Cuidado Paliativo, Acompañamiento en final del vida, Fisiatría, Medicina Física, Fisioterapia y Rehabilitación Oncológica.

Es necesario conocer las disposiciones de las Guías de Rehabilitación elaboradas por entidades competentes, para tal caso se consulta las guías elaboradas por el Colegio de Kinesiólogos de Chile (10), el Colegio de Terapeutas de Costa Rica (xvi), la World Phisioterapy (xvii) y la World Confederation For Physical Therapy (6) sobre el uso de las TIC's en Práctica Digital de la Salud, con el propósito de conocer las diferentes modalidades existentes.

Telerehabilitación Sincrónica: consiste en una sesión de tratamiento en tiempo real, entre un profesional de la salud y un paciente utilizando una plataforma o aplicación, por ejemplo, videoconferencia, monitoreo a distancia o tecnologías de juego virtual.

Telerehabilitación Asincrónica: es la transmisión diferida del material para diagnóstico y tratamiento con el fin de que el profesional de la salud lo pueda revisar y almacenar en un momento posterior al de la consulta directa, por ejemplo, video, fotos y correo electrónico.

> Telerehabilitación Híbrida: involucra tanto la modalidad sincrónica (en tiempo real) como la asincrónica (diferida) para la atención clínica. 
Teleconsulta: consulta a la distancia realizada a través de TICs entre un paciente y uno (o más) miembro(s) del equipo de salud ubicados en lugares geográficos distintos con la posibilidad de interactuar entre sí.

Teleinterconsulta: es el intercambio de información, opiniones y la generación de eventuales diagnósticos y decisiones terapéuticas sobre un caso clínico entre miembros del equipo de salud a distancia a través de TICs, sin la presencia o participación directa del paciente.

Telemonitoreo: se dice de la transmisión de información por medio de dispositivos de monitoreo que el paciente presenta instalados en su cuerpo o a disposición en su hogar, para el monitoreo del estado de salud o el establecimiento de un diagnóstico.

Teleconsultoría: se conoce como la comunicación registrada y llevada a cabo entre profesionales de la salud y otras profesiones afines, basada en evidencia y protocolos clínico-científicos, para aclarar dudas sobre procedimientos clínicos, acciones de salud y cuestiones relacionadas con el proceso.

Entre las modalidades que se encontraron en la presente revisión bibliográfica se destacan:

Telerehabilitación Sincrónica: llamadas telefónicas y videollamadas.

Telerehabilitción Asincrónica: video, fotos, mensajes de texto, correo electrónico.

Telerehabilitación Híbrida: combinación de llamadas y envío de material como vídeo por medios electrónicos.

Teleconsulta: videoconferencias entre el personal de servicio de salud y el paciente / cuidador.

Telemonitoreo: aplicaciones para celulares inteligentes en diferentes sistemas operativos para el cuidado general, para el manejo de la enfermedad crónica, control de dolor y calidad de vida, aplicación educativa en Cuidados Paliativo, aplicaciones en conversión de uso de medicamentos.

Para determinar el impacto de las TIC's en Práctica Digital de la Salud en personas con cáncer terminal se realiza en la discusión, un análisis FODA (fortalezas, oportunidades amenazas y debilidades) basada en las conclusiones y recomendaciones de los documentos consultados. Finalmente, se establece una serie de ideas 
y recomendaciones que se pueden implementar en TIC's en Práctica Digital de la Salud en Rehabilitación en personas con cáncer en estadio terminal.

\section{Discusión}

Actualmente, se sabe que a través de la telerehabilitación se pueden obtener resultados comparables con los programas de rehabilitación tradicionales (xviii).

Los estudios que relacionan cáncer con Telemedicina se centran en las fases de prevención, diagnóstico, toma de decisiones, tratamiento y seguimiento, tal como lo evidencia Nasi et al (xix), sin mencionar la Rehabilitación en Cuidados Paliativos y Acompañamiento al Final de la Vida tal como muestra la Figura N2.

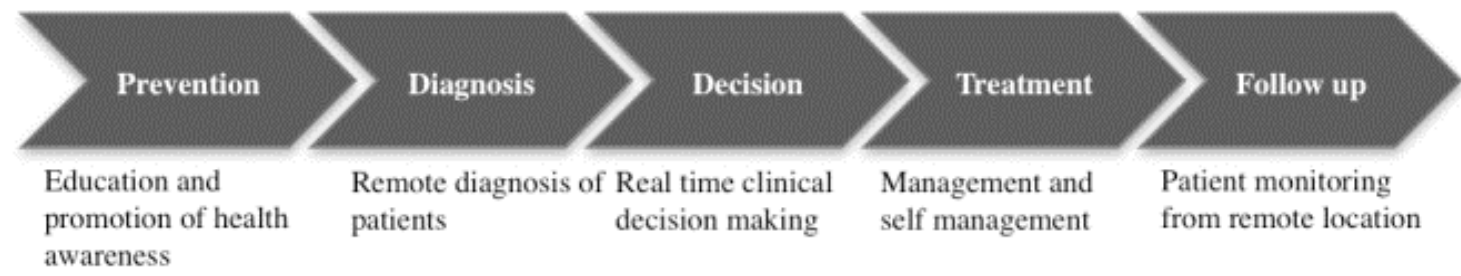

Figura N2 Tecnologías móviles en el proceso asistencial

Fuente: Nasi G, Cucciniello M, Guerrazzi C. The role of mobile technologies in health care processes: the case of cancer supportive care. J Med Internet Res. 2015 Feb 12;17(2):e26. doi: 10.2196/jmir.3757. PMID: 25679446; PMCID: PMC4342745.

Los Cuidados Paliativos y el Acompañamiento en Final de Vida se podrían ubicar en la fase del tratamiento y seguimiento, y utilizar la tecnología móvil para controlar los síntomas y la condición del usuario o, para permitir que el mismo o sus cuidadores, lo realicen por su cuenta (autogestión).

Entre los beneficios que esto implica, se encuentran poder tratar a pacientes con cáncer terminal en ubicaciones remotas (19) sin la necesidad de que estos se trasladen hasta el centro de salud y los riesgos y complicaciones que esto implica. Además de brindar una buena atención a estos pacientes, un objetivo para la Telemedicina en la fase terminal de cáncer es reducir las transferencias e ingresos innecesarios a los hospitales de agudos, permitiendo así que nuestros residentes mueran en su lugar preferido $\left.{ }^{(x}{ }^{2}\right)$. 
Durante los últimos años se han realizado esfuerzos para determinar el efecto de la Telemedicina en servicios de Rehabilitación. En el 2012 Mosa et al (xxi) revisa 55 artículos que reportaron 83 aplicaciones para celulares inteligentes que entre algunos otros temas se encontraban aplicaciones para comunicación clínica, aplicaciones de cuidado general, aplicaciones para las pacientes enfocadas en el manejo de la enfermedad crónica, aplicaciones relacionados con Enfermedades no Transmisibles, sin embargo, ninguna de ellas era orientada a la Rehabilitación Oncológica en fase terminal.

Para el 2018 según una búsqueda realizada en la base de datos del Instituto Nacional de Salud de EE.UU, existían ocho estudios en Fisioterapia que investigan la digital en dolor crónico, accidente cerebrovascular, esclerosis múltiple y artroplastia total de rodilla (18), por su parte Nwosu et al (xxii) revisaron en el 2012 las aplicaciones para teléfonos inteligentes dirigidas a profesionales de Cuidados Paliativos para cinco sistemas operativos, encontraron seis aplicaciones orientadas a blogs (Pallimed y Geripal), una aplicación que contiene pautas para ocho redes de cáncer (PalliApp), una aplicación educativa (Cuidados paliativos) y aplicaciones de conversión de dosis de opioides (eOpioid y PalliCalc).

Sin embargo, las investigaciones que abarquen el tema en Rehabilitación en Cuidados Paliativos en Cáncer en Estadio Terminal son escasas, y la mayoría se centran en la valoración o seguimiento del dolor y la calidad de vida mediante vía telefónica o teléfonos inteligentes, no así de métodos de rehabilitación para abordar el dolor, mejorar calidad de vida, movilizaciones, ejercicios, recomendaciones posturales, u otras complicaciones físicas ni dispositivos o el uso de TICs para estos abordajes.

Capurro D et al (xxii) en el 2013, realiza una revisión sistemática en Alemania, Perú y Chile, la cual deja ver que los pacientes de la zona urbana tenían mayores facilidades para acceder a los cuidados paliativos comparados con los que estaban en las zonas rurales. En el mismo año Zhoun et al (xxiv) en el 2013, investiga el efecto de la utilización de teléfonos en el seguimiento de pacientes de cuidados paliativos en cáncer de mama, de ovario, de próstata y glioma maligno, evidenciando que el monitoreo de los pacientes por medio del teléfono reduce significativamente la ansiedad y la depresión más que el seguimiento en la consulta presencial, además de reducir la carga presentada con los seguimientos clínicos y lleva al mejoramiento de la calidad de vida.

Tieman et al (xxv) estudia la viabilidad de un modelo basado en la telesalud en cuidados paliativos para los pacientes con cáncer avanzado remitidos por el Centro de Cuidados Paliativos del Hospital Universitario Pedro 
Ernesto en Rio de Janeiro (UERJ). El sistema se basa en videoconferencias entre el personal de servicio de salud y el paciente / cuidador. Los resultados obtenidos muestran que los pacientes y los cuidadores eran capaces de administrar la tecnología y proporcionar datos que de otro modo no estarían disponibles; además la tecnología había sido efectiva para asegurar que el paciente o cuidador fuera tranquilizado; así mismo, refirieren una identificación y manejo más rápido de los problemas y la capacidad de compartir información con otros profesionales de la salud. Por último, la calidad del contacto de telesalud era aceptable y en la mayoría de los casos comparada con los modos de contacto actuales.

En un estudio realizado en el 2015 por Charlton et al (xxvi) en Indiana Estados Unidos, concluye que la gestión centralizada en las llamadas por teléfono generaba mejorías significativas tanto en el dolor y la depresión de los pacientes con cáncer. Kidd (xxvii) realiza un estudio en un grupo de niños, en el cual se compararon los registros de consultas paliativas pediátricas realizadas cara a cara, con consultas de telemedicina realizadas en los hogares de los pacientes y confirmaron que las consultas paliativas a través de la telemedicina son tan efectivas como las consultas cara a cara en términos de los componentes documentados de la consulta.

La evidencia de la Telesalud en Cuidados Paliativos demuestra que estas técnicas parecen ser viables y no parecen agregar una carga adicional a la mayoría de los usuarios, ya que estos pueden sentirse más cómodos, en control y en un lugar más seguro en casa que en los hospitales (xxviii).A pesar de que la evidencia científica hasta ahora publicada ha demostrado que los resultados preliminares de las acciones de salud mediadas por las TIC's e implementada a través de diferentes modelos de intervención resultan ser valiosos para los pacientes en cuidados paliativos. Alvarez et al (xxix) menciona la relevancia de

\footnotetext{
i Ministerio de Salud Costa Rica. Plan Nacional para la Prevención y Control del Cáncer 2011- 2017. 1era edición. San José, Costa Rica. 2012.
}

iiOrganización Mundial de la Salud (OMS). http://www.who.int/cancer/palliative/es/

iii Capurro D, Ganzinger M, Perez-Lu J, Knaup P. Effectiveness of eHealth interventions and information needs in palliative care: A systematic literature review. J Med Internet Res [Internet]. 7 de marzo de 2014 [Acceso 24 de abril de 2017];16(3). Disponible en: http://www.ncbi.nlm.nih.gov/pmc/articles/PMC3961802/ 
iv Brenes, Brenes, Núñez: Pasado Y Presente De Los Cuidados Paliativos. Revista Médica De Costa Rica Y Centroamerica Lxx (605) 71-76, 2013

v Organización Mundial de la Salud, "Telemedicine Opportunities and developments in Members States. Report on the second global survey on eHealth. Global Observatory for eHealth series", Organización Mundial de la Salud, vol. 2, 2010.

vi WORLD CONFEDERATION FOR PHYSICAL THERAPY- International Network of Physiotherapy Regulatory AuthoritiesFebrero 24,2020.

vii E. Y. Parrasi Castaño, L. Celis Carvajal, J. J. Bocanegra García, Y. S. Pascuas Rengifo, "Estado actual de la telemedicina: una revisión de literatura". Ingeniare, NN. 20, pp. 105-120, 2016.

viii Andrés Fernández and Enrique Oviedo e-Health in Latin America and the Caribbean: progress and challenges. Salud-e (i) 2011.indd 3

ix Organización Panamericana de la Salud. Plan subregional para la prevención y control del cáncer en Centroamérica y República Dominicana. 2008 [ Links ]

× Colegio de kinesiologos de Chile. Guía de telerehabilitación para kinesiólogos - COLKINE. Versión 1.0 - 22 abril de 2020

xi Wenhua Liang, Weijie Guan, et al. Cancer patients in SARS-CoV-2 infection: a nationwide analysis in China Lancet. 2020; (publicado online 14 de febrero 2020.)

xii Dignani MC, Costantini P, Salgueira C et al. Pandemic 2009 influenza A (H1N1) virus infection in cancer and hematopoietic stem cell transplant recipients; a multicenter observational study. F1000Res 2014;3:221

xiii Yu J, Ouyang W, Chua MLK et al. SARS-CoV-2 transmission in cancer patients of a tertiary hospital in Wuhan. JAMA Oncol 2020 [Epub ahead of print].

xiv Al-Shamsi, Alhazzani, Alhuraiji et al. A Práctical Approach to the Management of Cancer Patients During the Novel Coronavirus Disease 2019 (COVID-19) Pandemic: An International Collaborative Group. The Oncologist 2020;25:e936-e945 www.The0ncologist.com 
xv Uclés Villalobos Vanessa, Mata Jiménez Laura, Matamoros Sánchez Arnoldo. Descripción de la población de personas con cáncer de mama referida al Programa de Rehabilitación en Cáncer y Cuidados Paliativos del Servicio de Fisiatría del Hospital Dr. Rafael Ángel Calderón Guardia, 01 de junio del 2012 al 31 de mayo del 2013. Rev. costarric. salud pública [Internet]. 2017 June [cited 2021 Mar 12] ; 26( 1 ): 30-44. Available from: http://www.scielo.sa.cr/scielo.php?script=sci_arttext\&pid=S1409-14292017000100030\&lng=en.

xvi Lineamiento Generales para el Ejercicio de la Telesalud. Colegio de Terapeutas de Costa Rica, 2020.

xvii INPTRA. Resources Digital Practice White Paper and Survey http://www.inptra.org/Resources/DigitalPracticeWhitePaperandSurvey.aspx

xviii Kairy D, Lehoux P, Vincent C, Visintin M. A systematic review of clinical outcomes, clinical process, healthcare utilization and costs associated with telerehabilitation. Disabil Rehabil.2009;31(6):427-47.

xix Nasi G, Cucciniello M, Guerrazzi C. The role of mobile technologies in health care processes: the case of cancer supportive care. J Med Internet Res. 2015 Feb 12;17(2):e26. doi: 10.2196/jmir.3757. PMID: 25679446; PMCID: PMC4342745.

xx . Low JA, Beins G, Lee KK, Koh E. Last moments of life: can telemedicine play a role? Palliat Support Care 2013;11:353-5. doi: 10.1017/S1478951512000995.

xxi Mosa ASM, Yoo I, Sheets L. A systematic review of healthcare applications for smartphones. BMC Med Inform Decis Mak 2012;12:67. doi: 10.1186/1472-6947-12-67

xxii Nwosu AC, Mason S. Palliative medicine and smartphones: An opportunity for innovation? BMJ Support Palliat Care. 2012;2:75-7.

xxiii Capurro D, Ganzinger M, Pérez-Lu JE. Palliative care from a medical informatics perspective in Chile, Germany, and Peru. Stud Health Technol Inform 2013;192:1013. doi: 10.3233/978-1-61499-289-9-1013

xxiv Zhou M, Holden L, Bedard G, Zeng L, Lam H, Chu D, et al. The utilization of telephone follow-up in the advanced cancer population: a review of the literature. J Comp Eff Res 2012;1:509-17. doi: 10.2217/cer.12.63 xxv Tieman JJ, Swetenham K, Morgan DD, To TH, Currow DC. Using telehealth to support end of life care in the community: a feasibility study. BMC Palliat Care 2016;15:94. Doi: 10.1186/s12904-016-0167-7. 
xxvi Charlton M, Schlichting J, Chioreso C, Ward M, Vikas P. Challenges of rural cancer care in the United States. Oncol Williston Park 2015;29:633-40.

xxvii Kidd L, Cayless S, Johnston B, Wengstrom Y. Telehealth in palliative care in the UK: a review of the evidence. J Telemed Telecare 2010;16:394-402. doi: 10.1258/jtt.2010.091108

xxviii Steindal SA, Nes AAG, Godskesen TE, Dihle A, Lind S, Winger A, Klarare A. Patients' Experiences of Telehealth in Palliative Home Care: Scoping Review. J Med Internet Res. 2020 May 5;22(5):e16218. doi: 10.2196/16218. PMID: 32369037; PMCID: PMC7238080.

xxix Alvarez-Tobón VA, Luna-Gómez IF, Torres-Silva EA, Higuita-Úsuga A, Rivera-Mejía PT. Tecnologías de información y comunicación (TIC) aplicadas en cuidados paliativos: revisión de tema. Psicooncología 2018;15:345-360. doi: 10.5209/PSIC.61440. del contacto "cara a cara", ya que este ha resultado importante tanto para los pacientes como para los profesionales y hacer énfasis en que la Telemedicina es complemento y no remplazo.

Se presenta seguidamente el Análisis FODA del uso de TIC's en Práctica Digital de la Salud en personas con cáncer terminal, con el objetivo de entender a mayor cabalidad las facilidades y oportunidades para lograr disminuir el efecto de las debilidades y amenazas del uso de las TIC's en la población estudiada y de esta manera presentar una serie de recomendaciones e ideas para las personas con cáncer terminal. 


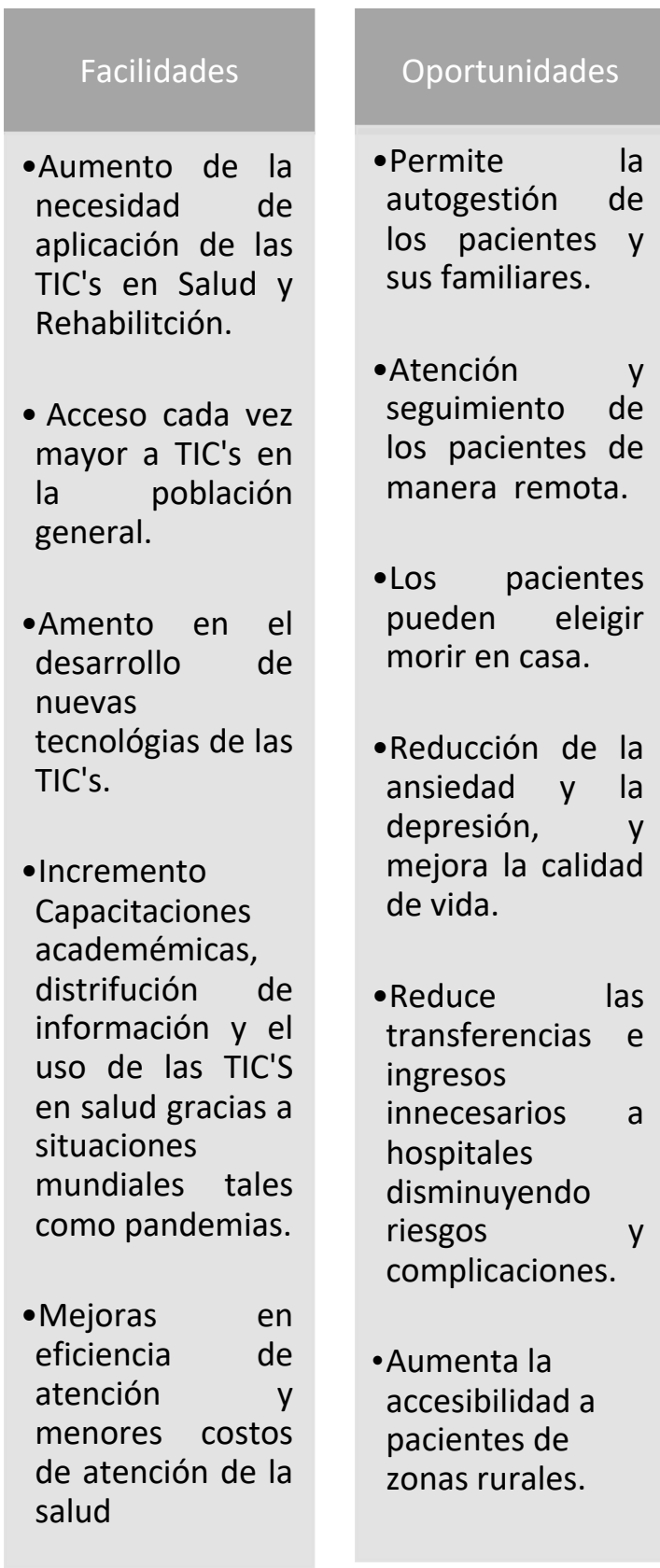

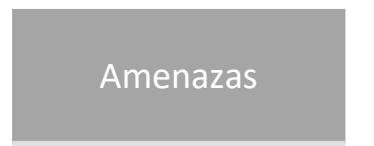

-Acceso desigual a TICS entre los pacientes y sus familias.

- Falta de formación por parte de los profesionales, pacientes familias para el uso de las TIC's en salud.

- Escases de investigación de TICs en Rehabilitación en Cuidados Paliativos en Cáncer en Estadio Terminal.

\section{Debilidades}

- La evidencia cinetifica actual se centra en unicamente la valoración o seguimiento del dolor y la calidad de vida, no así en ejericicos, movilizaciones, demás.

- Respuesta de personal de salud en caso de emergencia comprometida por distancia.

-Afecetacción del efecto del contacto "cara a cara" durante la atención personal

Figura N3 Análisis FODA de la aplicación de las TIC's en Práctica Digital de la Salud en Rehabilitación en personas con cáncer en estadio terminal.

Fuente: Elaboración propia a partir de la bibliografía consultada. ${ }^{3-45 .}$

Observando los beneficios que poseen la TIC's en Práctica Digital de la Salud en Cuidados Paliativos y Cáncer en estadio Terminal, surge la cuestionante que todos los profesionales de la salud hemos tenido que enfrentar 
con mayor frecuencia y severidad en los últimos años: Cómo se podrían aplicar las TICs en el abordaje de Rehabilitación en las personas con Cáncer en estadio Terminal.

Una solución viable podría ser tomar como referencia TICs utilizados en otras situaciones de la salud semejantes, de alguna manera, a las condiciones, patologías u objetivos de abordaje en nuestra población meta, de esta manera se podrían elaborar nuevos modelos adaptados a las necesidades y características a las personas con cáncer terminal que sirvan como opciones abordaje de la Rehabilitación en el fin de la vida destacando temas como el autocuidado tanto de la persona con cáncer como el de los cuidadores, seguimiento de ejercicios, masaje oncológico, manipulaciones, movilizaciones, cambios posturales, uso de dispositivos portátiles de agentes electro físicos como el TENS, educación de temas relacionados, entre otros, mediante vía remota.

Dado el auge de la portabilidad y los teléfonos celulares smartphones se vislumbra una oportunidad para el abordaje de los pacientes como acciones de TIC's en Práctica Digital de la Salud en Cáncer en estadio terminal $($ xxix), son una de las estrategias que se podrían aplicar fácilmente ya que pueden resolver varios de los principales problemas que se encuentran en los países de bajos ingresos: distancia, acceso limitado a las computadoras y falta de trabajadores de la salud, lo que permite mejoras en términos de eficiencia y menores costos de atención de la salud (xxix). Según Bender (xxix), los teléfonos inteligentes y las aplicaciones móviles son la tecnología más utilizada en la atención de personas con cáncer tanto en su detección, tratamiento y seguimiento.

Kearney (xxix) desarrolla un Sistema Avanzado de Gestión de Síntomas (ASyMSA) en donde los pacientes deben completar un cuestionario electrónico para registrar y enviar informes de síntomas diarios al centro de atención oncológica e inmediatamente reciben asesoramiento instantáneo y personalizado por escrito a través de la interfaz del teléfono móvil sobre el manejo de los síntomas basado en un ciclo de dos tratamientos.

Las tecnologías como los sensores, mecanismos de captura de movimientos óptico, robots, Wearables, entre otros dispositivos permiten la rehabilitación del paciente y la transmisión de los resultados obtenidos a un centro médico especializado (xxix). Domb et al (xxix) menciona que la nueva generación de sensores portátiles permite a los profesionales de la salud capturar los niveles de actividad de los pacientes a largo plazo y el cumplimiento del ejercicio, lo que facilita la dispensación eficaz de tratamiento para pacientes crónicos y proporciona herramientas para evaluar su capacidad para realizar actividades motoras específicas y proponer 
soluciones de rehabilitación. Por lo tanto, su aplicación en personas con cáncer terminal podría ser de gran utilidad.

En la actualidad, no existen recomendaciones específicas para el uso de Wearables en oncología, y pocas investigaciones han examinado el propósito del uso de wearables en esta área. Por lo cual se alienta la investigación que utilice dispositivos portátiles avanzados y el uso activo de los datos para explorar más a fondo el potencial de los dispositivos portátiles en oncología en fase terminal. Pero se podrían utilizar los existentes para medir los rangos de movilidad articular, frecuencia cardiaca, saturación de oxígeno, presión arterial y otras variables necesarias para la prescripción de ejercicio oncológico y técnicas como manipulaciones y movilizaciones.

Stinton et al (xxix) desarrolla Pain Squad para iPhone para abordar el problema del dolor mal gestionado en el grupo de adolescentes con cáncer. La aplicación es un diario electrónico del dolor y recopila datos sobre la intensidad del dolor, la duración, la ubicación y el impacto que tiene el dolor en la vida de un adolescente (es decir, relaciones, trabajo escolar, sueño, estado de ánimo). También evalúa los medicamentos y otras estrategias de manejo del dolor físico y psicológico utilizadas, concluyendo que es un método válido y confiable con capacidades de manejo del dolor tiene la capacidad de resultar en un mejor manejo del dolor y, en última instancia, mejorar la calidad de vida de los adolescentes con cáncer

Pavic et al (xxix) realiza un estudio entre Febrero del 2017 a mayo del 2019 donde se incluyeron treinta pacientes mayores de 18 años con cáncer y una esperanza de vida de pronóstico limitado a menor de un año de vida, a los cuales se les proporcionó un teléfono inteligente, incluida la aplicación "Monitorización de actividad" preinstalada y un brazalete equipado con un sensor, los cuales registran la frecuencia cardiaca en reposo, velocidad de pasos, la escala analógica visual (EVA) para el dolor y la angustia y, el Cuestionario de Calidad de Vida del Cáncer-Core 30 (EORTC QLQ-C30). Entre las conclusiones se destaca que el seguimiento de los pacientes con cáncer paliativo mediante Wearables es factible. Los primeros resultados indican que Las características de salud móvil podrían ser biomarcadores prometedores para predecir readmisiones no planificadas.

Por ejemplo, Brewerr et al (xxix) elaboró un Programa que crea un entorno virtual donde interactúan el paciente cardiaco y especialista en sesiones bajo las cuales se realizan las terapias cardiacas, además, el sistema cuenta con un módulo de vida saludable, en el que se indica al paciente la dieta que debe llevar y los 
ejercicios que debe realizar, con el objetivo de implantar en la persona un estilo de vida sana, y así reducir los padecimientos coronarios. Siguiendo esta línea, se podría elaborar un emulador (simulador) para la ejecución de ejercicio terapéutico oncológico, masaje oncológico, movilizaciones articulares pasivas, ya sea activo o asistido, y entre otras técnicas, con el fin de mantener la funcionalidad, evitar en lo posible las adherencias en articulaciones y la atrofia.

Hochstenbach en el 2016 (xxix) menciona que los pacientes paliativos que tendían a olvidar qué medicación habían tomado, consideraron la descripción general de la medicación en una Aplicación de Recordatorios de horas y medicamentos, como un apoyo, y aquellos que tomaron sus analgésicos en función del reloj de la aplicación encontraron útiles los recordatorios visuales y sonoros de la misma. De la misma manera, se podría elaborar una aplicación que genera una descripción de la movilización pasiva, el ejercicio físico oncológico, o el cambio de posturas, y los recordatorios a la hora recomendada para realizarlos.

Steindal et al (28) en el 2020 realiza una Revisión Sistemática, donde menciona que las personas en cuidados paliativos que experimentaron la mediante alguna modalidad de vídeo, encontraban que la técnica era útil para la comunicación y la interacción con los profesionales de la salud, ya que los pacientes notaron que las características visuales les permitían ver a los profesionales de la salud con los que estaban interactuando, lo que les brindaba seguridad y comodidad, así como una mejor atención y evaluaciones. Además, el uso de vídeo permite la comunicación no verbal, como el lenguaje corporal, las expresiones faciales de felicidad o sufrimiento y otras emociones, además del contexto situacional (xxix, xxix).

Es de suma importancia tener claro que este tipo de herramientas son solamente un apoyo, no un sustituto de la atención presencial. En un estudio elaborado por Reinke et al (xxix) en donde se entrenan a los pacientes sobre la comunicación al final de la vida mediante seminarios web, estos informaron que este formato carecía de dinámicas interpersonales como la presencia social, así como señales de comunicación auditiva y visual que se consideraban importantes debido a la naturaleza sensible del tema. Además, los pacientes afirmaron que tener el video en vivo del facilitador de la discusión y la capacidad de ver a otros participantes podría haber facilitado el seguimiento de las discusiones.

Antes de elaborar alguna de estas modalidades de TIC's en Cáncer en fase terminal, se debe considerar algunos requisitos previos para que las personas objetivo se encuentren dispuestos a utilizar la telesalud. En este caso Johnston et al ( $\left.{ }^{x i x}\right)$. afirma que la simplicidad de las aplicaciones y la percepción de que los servicios 
de telesalud son valiosos para los pacientes son indispensables, además considera que los problemas de diseño de las aplicaciones elaborada de manera inadecuada podrían traer consecuencias negativas de usabilidad y dificultar el uso para algunas personas.

Para que estas herramientas brindar mejores resultados y experiencias, se sugiere que los profesionales de la salud que brindan cuidados paliativos y laboren en acompañamiento al final de la vida, trabajen conjuntamente con los profesionales que hacen el software para desarrollar dichas herramientas (30) y así obtener aplicaciones adecuadas a las características biopsicosociales y las necesidades de la familia, los cuidadores y los pacientes (xxix).

Lo anterior ya que los problemas de diseño pueden aumentar la dependencia de la ayuda de otros o actuar como un recordatorio del deterioro de la salud y la muerte inminente. Para optimizar el diseño de las TIC's en este campo, se recomienda la participación de los usuarios en el proceso de diseño de soluciones técnicas, con el propósito de aplicaciones y servicios de telesalud que estén aún más alineados con las necesidades del paciente (xxix).

Se presenta una serie de ideas para el desarrollo de TIC's en Práctica Digital de la Salud en Cuidados Paliativos y Cáncer en estadio Terminal.

\begin{tabular}{|c|c|}
\hline TIC's & Funciones \\
\hline $\begin{array}{l}\text { Herramienta para el } \\
\text { monitoreo, control y } \\
\text { recomendaciones de } \\
\text { síntomas y signos. }\end{array}$ & $\begin{array}{l}\text { Desarrollar una herramienta que permita el acceso a los pacientes y/o familiares a } \\
\text { Instrumentos y/o cuestionarios electrónicos, para registrar y enviar informes de } \\
\text { síntomas y sinos diarios y/o semanales. } \\
\text { Debe permitir retroalimentación mediante el envió de asesoramiento personalizado } \\
\text { por texto o videos mediante electrónicas de comunicación. }\end{array}$ \\
\hline
\end{tabular}




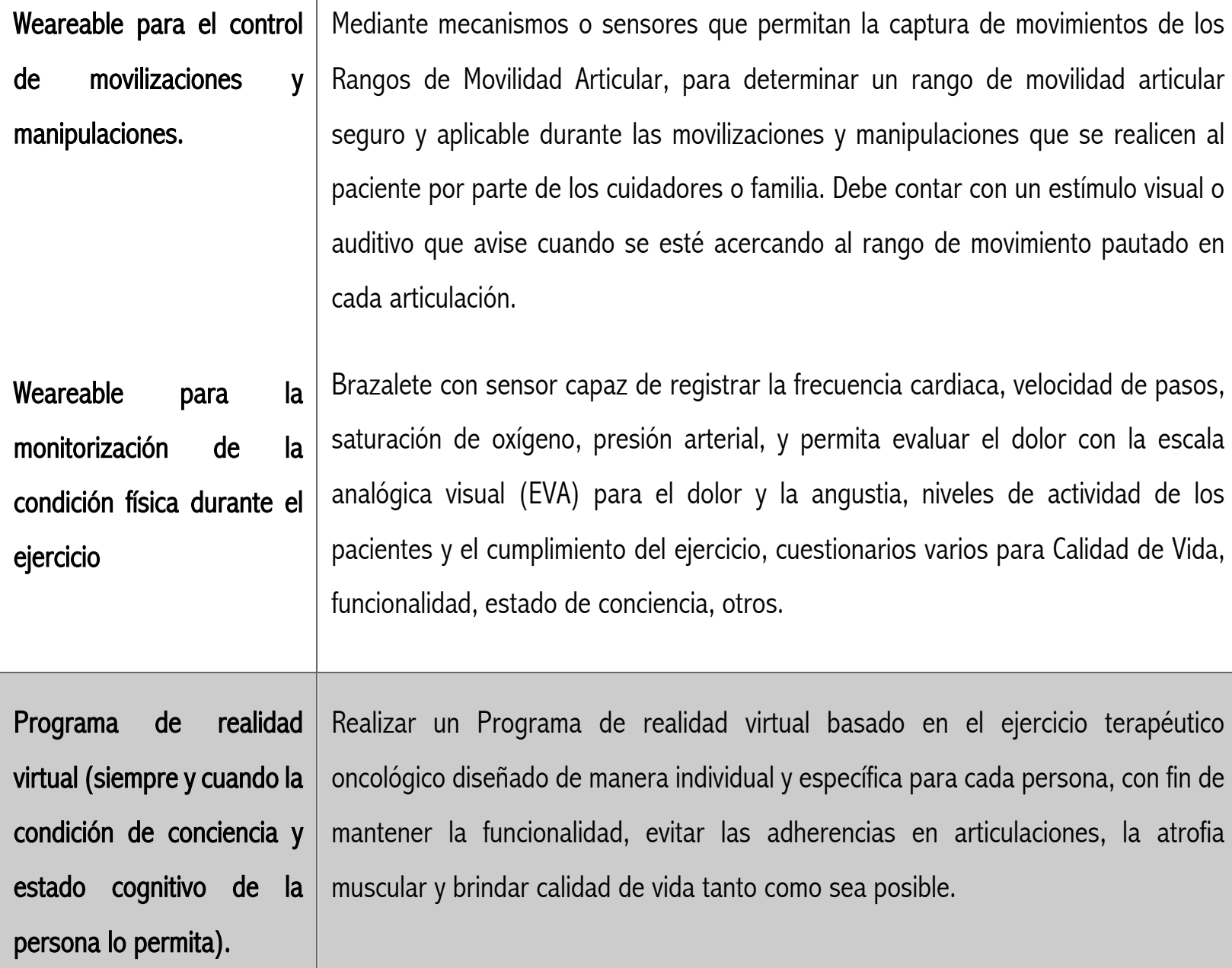

Weareable para la Brazalete con sensor capaz de registrar la frecuencia cardiaca, velocidad de pasos, monitorización de la condición física durante el ejercicio analógica visual (EVA) para el dolor y la angustia, niveles de actividad de los pacientes y el cumplimiento del ejercicio, cuestionarios varios para Calidad de Vida, funcionalidad, estado de conciencia, otros.

Programa de realidad Realizar un Programa de realidad virtual basado en el ejercicio terapéutico virtual (siempre y cuando la oncológico diseñado de manera individual y específica para cada persona, con fin de condición de conciencia y mantener la funcionalidad, evitar las adherencias en articulaciones, la atrofia estado cognitivo de la muscular y brindar calidad de vida tanto como sea posible. persona lo permita).

\section{Diario electrónico}

Aplicación de acceso en dispositivo móvil que permita evaluar el dolor y recopilar datos sobre su intensidad, la duración, la ubicación y el impacto que tiene el dolor en la vida. Con los datos recopilados los profesionales de salud pueden elaborar estrategias para mejorar la condición de la persona. 


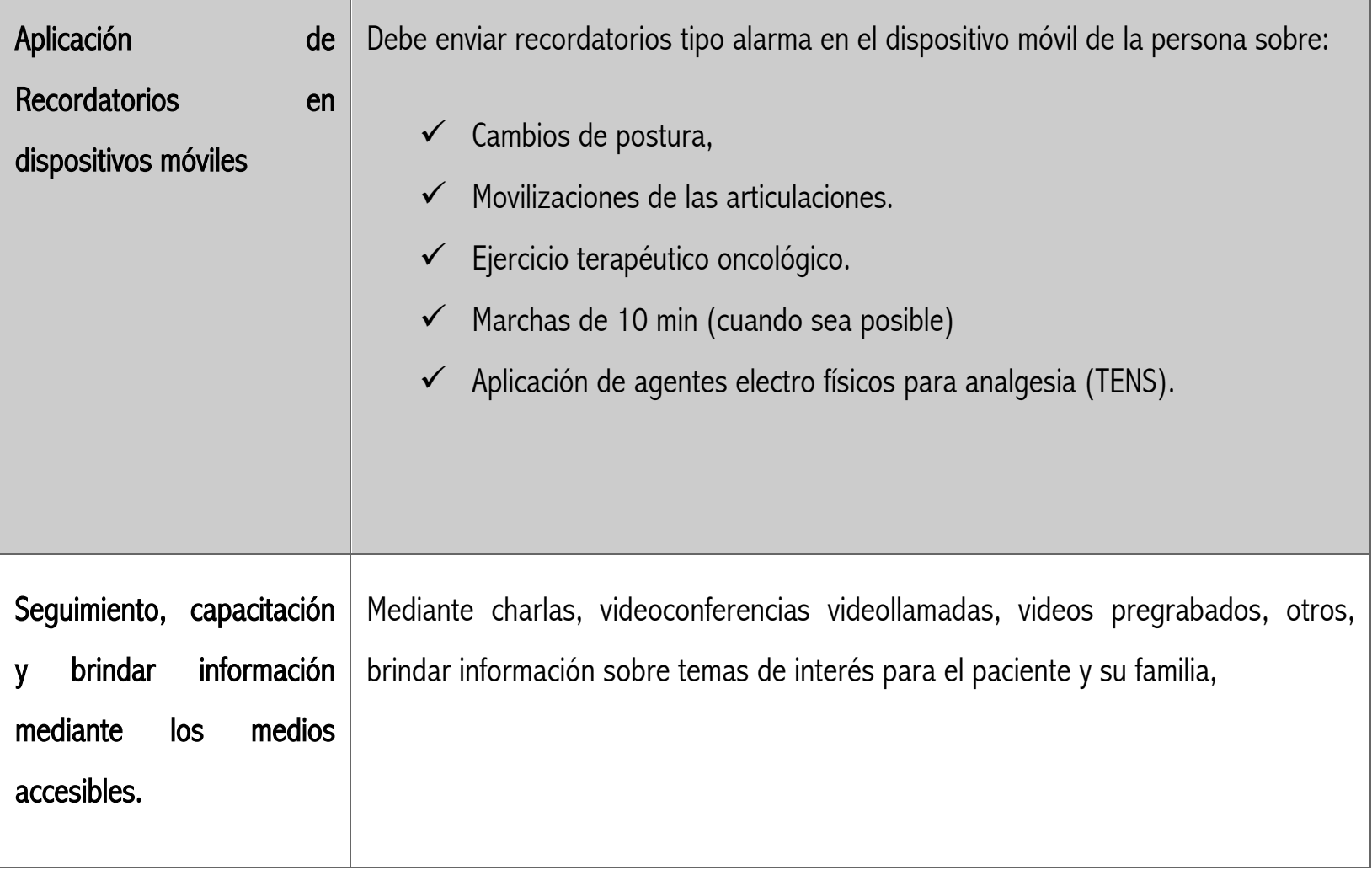

Figura N4. Ideas para el desarrollo de TIC's en Práctica Digital de la Salud en Cuidados Paliativos y Cáncer en estadio Terminal.

Fuente: Elaboración propia a partir de la bibliografía consultada 3-45.

\section{Conclusiones}

Las TIC's orientados a la Rehabilitación Oncológica en estadio terminal podría tener un futuro prometedor ya que se ha evidenciado sus múltiples beneficios y alcances positivos en otras situaciones de la salud. Se ha visto beneficios como aumento de la calidad de vida, control del dolor, depresión, ansiedad, disminución de riesgo asociados al traslado a hospitales, aumento del alcance de atención a personas de zonas de difícil acceso, entre otros. Además, las limitaciones del uso de estas tecnologías pueden tener soluciones viables y plausibles como la distribución o préstamo de accesorios necesarios (celulares, Tabletas, otros), capacitación de los profesionales de la salud, paciente y familiares, desarrollo de TIC's acorde a la población, entre otros. Para elaborar estas tecnologías, es necesario el trabajo en conjunto con profesionales de la salud, profesionales en desarrollo tecnologías, pacientes y familia para lograr TIC's efectivas y viables en Práctica Digital de la Salud en Cáncer en estadio terminal. Se recomienda el desarrollo de investigaciones futuras en el desarrollo y uso de 
TIC's en Práctica Digital de la Salud en Cáncer en estadio terminal, para lograr determinar de mejor manera su impacto en esta población.

\section{Otros}

Declaración de Conflictos de Interés: La autora declara que no existen conflictos de interés.

Agradecimientos: A la Dra. Vanesa Uclés por ser pionera en la Rehabilitación Oncológica en Costa Rica, por la dedicación y pasión que dedica en su trabajo y por compartir el conocimiento adquirido con los demás profesionales de la rehabilitación

\section{Referencias}

Ministerio de Salud Costa Rica. Plan Nacional para la Prevención y Control del Cáncer 2011- 2017. 1era edición. San José, Costa Rica. 2012.

xxixOrganización Mundial de la Salud (OMS). http://www.who.int/cancer/palliative/es/

xxix Capurro D, Ganzinger M, Perez-Lu J, Knaup P. Effectiveness of eHealth interventions and information needs in palliative care: A systematic literature review. J Med Internet Res [Internet]. 7 de marzo de 2014 [Acceso 24 de abril de 2017];16(3). Disponible en: http://www.ncbi.nlm.nih.gov/pmc/articles/PMC3961802/

xxix Brenes, Brenes, Núñez: Pasado Y Presente De Los Cuidados Paliativos. Revista Médica De Costa Rica Y Centroamerica Lxx (605) 71-76, 2013

xxix Organización Mundial de la Salud, "Telemedicine Opportunities and developments in Members States. Report on the second global survey on eHealth. Global Observatory for eHealth series», Organización Mundial de la Salud, vol. 2, 2010.

xxix WORLD CONFEDERATION FOR PHYSICAL THERAPY- International Network of Physiotherapy Regulatory AuthoritiesFebrero 24,2020.

xxix E. Y. Parrasi Castaño, L. Celis Carvajal, J. J. Bocanegra García, Y. S. Pascuas Rengifo, "Estado actual de la telemedicina: una revisión de literatura". Ingeniare, N. 20, pp. 105-120, 2016. 
xxix Andrés Fernández and Enrique Oviedo e-Health in Latin America and the Caribbean: progress and challenges. Salud-e (i) 2011.indd 3

xxix Organización Panamericana de la Salud. Plan subregional para la prevención y control del cáncer en Centroamérica y República Dominicana. 2008 [ Links ]

xxix Colegio de kinesiologos de Chile. Guía de telerehabilitación para kinesiólogos - COLKINE. Versión 1.0 - 22 abril de 2020

xxix Wenhua Liang, Weijie Guan, et al. Cancer patients in SARS-CoV-2 infection: a nationwide analysis in China Lancet. 2020; (publicado online 14 de febrero 2020.)

xxix Dignani MC, Costantini P, Salgueira C et al. Pandemic 2009 influenza A (H1N1) virus infection in cancer and hematopoietic stem cell transplant recipients; a multicenter observational study. F1000Res 2014;3:221

xxix Yu J, Ouyang W, Chua MLK et al. SARS-CoV-2 transmission in cancer patients of a tertiary hospital in Wuhan. JAMA Oncol 2020 [Epub ahead of print].

xxix Al-Shamsi, Alhazzani, Alhuraiji et al. A Práctical Approach to the Management of Cancer Patients During the Novel Coronavirus Disease 2019 (COVID-19) Pandemic: An International Collaborative Group. The Oncologist 2020;25:e936-e945 www.TheOncologist.com

xxix Uclés Villalobos Vanessa, Mata Jiménez Laura, Matamoros Sánchez Arnoldo. Descripción de la población de personas con cáncer de mama referida al Programa de Rehabilitación en Cáncer y Cuidados Paliativos del Servicio de Fisiatría del Hospital Dr. Rafael Ángel Calderón Guardia, 01 de junio del 2012 al 31 de mayo del 2013. Rev. costarric. salud pública [Internet]. 2017 June [cited 2021 Mar 12] ; 26( 1 ): 30-44. Available from: http://www.scielo.sa.cr/scielo.php?script=sci_arttext\&pid=S1409-14292017000100030\&lng=en.

xxix Lineamiento Generales para el Ejercicio de la Telesalud. Colegio de Terapeutas de Costa Rica, 2020.

xxix INPTRA. Resources Digital Practice White Paper and Survey http://www.inptra.org/Resources/DigitalPracticeWhitePaperandSurvey.aspx

xxix Kairy D, Lehoux P, Vincent C, Visintin M. A systematic review of clinical outcomes, clinical process, healthcare utilization and costs associated with telerehabilitation. Disabil Rehabil.2009;31(6):427-47. 
xxix Nasi G, Cucciniello M, Guerrazzi C. The role of mobile technologies in health care processes: the case of cancer supportive care. J Med Internet Res. 2015 Feb 12;17(2):e26. doi: 10.2196/jmir.3757. PMID: 25679446; PMCID: PMC4342745.

xxix . Low JA, Beins G, Lee KK, Koh E. Last moments of life: can telemedicine play a role? Palliat Support Care 2013;11:353-5. doi: 10.1017/S1478951512000995.

xxix Mosa ASM, Yoo I, Sheets L. A systematic review of healthcare applications for smartphones. BMC Med Inform Decis Mak 2012;12:67. doi: 10.1186/1472-6947-12-67

xxix Nwosu AC, Mason S. Palliative medicine and smartphones: An opportunity for innovation? BMJ Support Palliat Care. 2012;2:75-7.

xxix Capurro D, Ganzinger M, Pérez-Lu JE. Palliative care from a medical informatics perspective in Chile, Germany, and Peru. Stud Health Technol Inform 2013;192:1013. doi: 10.3233/978-1-61499-289-9-1013

xxix Zhou M, Holden L, Bedard G, Zeng L, Lam H, Chu D, et al. The utilization of telephone follow-up in the advanced cancer population: a review of the literature. J Comp Eff Res 2012;1:509-17. doi: 10.2217/cer.12.63

xxix Tieman JJ, Swetenham K, Morgan DD, To TH, Currow DC. Using telehealth to support end of life care in the community: a feasibility study. BMC Palliat Care 2016;15:94. Doi: 10.1186/s12904-016-0167-7.

xxix Charlton M, Schlichting J, Chioreso C, Ward M, Vikas P. Challenges of rural cancer care in the United States. Oncol Williston Park 2015;29:633-40.

xxix Kidd L, Cayless S, Johnston B, Wengstrom Y. Telehealth in palliative care in the UK: a review of the evidence. J Telemed Telecare 2010;16:394-402. doi: 10.1258/jtt.2010.091108

xxix Steindal SA, Nes AAG, Godskesen TE, Dihle A, Lind S, Winger A, Klarare A. Patients' Experiences of Telehealth in Palliative Home Care: Scoping Review. J Med Internet Res. 2020 May 5;22(5):e16218. doi: 10.2196/16218. PMID: 32369037; PMCID: PMC7238080. 
xxix Alvarez-Tobón VA, Luna-Gómez IF, Torres-Silva EA, Higuita-Úsuga A, Rivera-Mejía PT. Tecnologías de información y comunicación (TIC) aplicadas en cuidados paliativos: revisión de tema. Psicooncología 2018;15:345-360. doi: 10.5209/PSIC.61440.

xxix Littman-Quinn R, Chandra A, Schwartz A, Fadlelmola FM, Ghose S, Luberti AA, Tatarsky A, Chihanga S, Ramogola-Masire D, Steenhoff A, Kovarik C. mHealth applications for telemedicine and public health intervention in Botswana. 2011 IST-Africa Conference; 11-13 May 2011; Gaborone. 2011. pp. 1-11. [Google Scholar]

xxix Bender JL, Yue RY, To MJ, Deacken L, Jadad AR. A lot of action, but not in the right direction: systematic review and content analysis of smartphone applications for the prevention, detection, and management of cancer. J Med Internet Res. 2013;15(12):e287. doi: 10.2196/jmir.2661. http://www.jmir.org/2013/12/e287/ [PMC free article] [PubMed] [CrossRef] [Google $\underline{\text { Scholar] }}$

xxixKearney N, Hargan I, Miller M, Muir L, Gray P. The use of web based information in handheld computers in supporting patients receiving outpatient chemotherapy. European Journal of Cancer Supplements. 2003 Sep;1(5):S369. doi: 10.1016/S1359-6349(03)91235-3. [CrossRef] [Google Scholar]

xxix M. Callejas Cuervo, G. Diaz y A. F. Ruiz Olaya, «Integration of emerging motion capture technologies and videogames for human upper-limb telerehabilitation: A systematic review», DYNA Journal of the Facultad de Minas, vol. 82, 2014

xxix Domb, Menachem. (2019). Wearable Devices and their Implementation in Various Domains. 10.5772 /intechopen.86066.

xxix Stinson, Jennifer \& Jibb, Lindsay \& Nguyen, Cynthia \& Nathan, Paul \& Maloney, Anne \& Dupuis, Lee \& Gerstle, J \& Alman, Benjamin \& Hopyan, Sevan \& Strahlendorf, Caron \& Portwine, Carol \& Johnston, Donna \& Orr, Mike. (2013). Development and Testing of a Multidimensional iPhone Pain Assessment Application for Adolescents with Cancer. Journal of medical Internet research. 15. e51. 10.2196/jmir.2350.

xxix Matea Pavic, Vanessa Klaas, Gudrun Theile, Johannes Kraft, Gerhard Tröster, David Blum, and Matthias Guckenberger.Journal of Palliative Medicine.May 2020.678-685.http://doi.org/10.1089/jpm.2019.0342 
xxix L. Brewer, B. Kaihoi, K. Zarling, R. Squires, R. Thomas y S. Kopecky, «The Use of Virtual World-Based Cardiac Rehabilitation to Encourage Healthy Lifestyle Choices Among Cardiac Patients: Intervention Development and Pilot Study Protocol», JMIR RESEARCH PROTOCOLS, vol. 4, n² 2, 2015.

xxix Hochstenbach LM, Zwakhalen SM, Courtens AM, van Kleef M, de Witte LP. Feasibility of a mobile and webbased intervention to support self-management in outpatients with cancer pain. Eur J Oncol Nurs. 2016 Aug;23:97-105. doi: 10.1016/j.ejon.2016.03.009. https://linkinghub.elsevier.com/retrieve/pii/S14623889(16)30027-8. [PubMed] [CrossRef] [Google Scholar]

xxixVan Gurp J, van Selm M, Vissers K, van Leeuwen E, Hasselaar J. How outpatient palliative care teleconsultation facilitates empathic patient-professional relationships: a qualitative study. PLOS One. 2015;10(4):e0124387. doi: $\quad$ 10.1371/journal.pone.0124387. http://dx.plos.org/10.1371/journal.pone.0124387. [PMC free article] [PubMed] [CrossRef] [Google Scholar]

xxix Hennemann-Krause L, Lopes AJ, Araújo JA, Petersen EM, Nunes RA. The assessment of telemedicine to support outpatient palliative care in advanced cancer. Palliat Support Care. 2015 Aug;13(4):1025-30. doi: 10.1017/S147895151400100X. [ubMed] [CrossRef] [Google Scholar]

xxix Reinke LF, Griffith RG, Wolpin S, Donesky-Cuenco D, Carrieri-Kohlman V, Nguyen HQ. Feasibility of a webinar for coaching patients with chronic obstructive pulmonary disease on end-of-life communication. Am J Hosp Palliat Care. $2011 \quad$ May;28(3):147-52. doi: 10.1177/1049909110376807. http://europepmc.org/abstract/MED/20834035. [PMC free article] [PubMed] [CrossRef] [ Google Scholar]

xxix Johnston B, Kidd L, Wengstrom Y, Kearney N. An evaluation of the use of Telehealth within palliative care settings across Scotland. Palliat Med.2012 Mar;26(2):152-61. doi: 10.1177/0269216311398698. [PubMed] [CrossRef] [Google Scholar]

xxix Jamwal NR, Kumar SP. Smarter palliative care for cancer: Use of smartphone applications. Indian J Palliat Care 2016;22:108-10. doi: 10.4103/0973-1075.173950

xxix Clemensen J, Rothmann MJ, Smith AC, Caffery ப, Danbjorg DB. Participatory design methods in telemedicine research. J Telemed Telecare. 2017 Oct;23(9):780-5. doi: 10.1177/1357633X16686747. [PubMed] [CrossRef] [Google Scholar] 
